\title{
Influence of Data Scaling and Normalization on Overall Neural Network Performances in Photoacoustics
}

Miroslava Ivko Jordovic Pavlovic ( $\sim$ miroslava.jordovic-pavlovic@vpts.edu.rs )

Western Serbia Academy of Applied Studies https://orcid.org/0000-0003-4038-6537

Katarina Djordjevic

Institut za nuklearne nauke Vinča: Univerzitet u Beogradu Institut za nuklearne nauke Vinca

\section{Zarko Cojbasic}

Univerzitet v Nisu Masinski fakultet

\section{Slobodanka Galovic}

Institut za nuklearne nauke Vinča: Univerzitet u Beogradu Institut za nuklearne nauke Vinca

\section{Marica Popovic}

Institut za nuklearne nauke Vinča: Univerzitet u Beogradu Institut za nuklearne nauke Vinca

\section{Mioljub Nesic}

Institut za nuklearne nauke Vinča: Univerzitet u Beogradu Institut za nuklearne nauke Vinca

\section{Dragan Markushev}

Institute of Physics Belgrade: Univerzitet u Beogradu Institut za Fiziku

\section{Research Article}

Keywords: photoacoustic, semiconductors, artificial neural networks, thermal diffusion, thermal expansion, photothermal, inverse problem

Posted Date: October 18th, 2021

DOl: https://doi.org/10.21203/rs.3.rs-942309/v1

License: (c) (i) This work is licensed under a Creative Commons Attribution 4.0 International License. Read Full License 


\title{
Influence of data scaling and normalization on overall neural network performances in photoacoustics
}

\author{
K Lj Djordjevic ${ }^{1}$, M I Jordović-Pavlović 2 Ž M Ćojbašić ${ }^{3}$, S P Galović ${ }^{1}$, \\ M N Popović ${ }^{1}$, M V Nešić 1 , D D Markushev ${ }^{4}$ \\ ${ }^{1}$ University of Belgrade, Vinca Institute of Nuclear Sciences -National Institute of the Republic of Serbia, Belgrade, \\ Serbia \\ ${ }^{2}$ Western Serbia Academy of Applied Studies, Užice, Trg Svetog Save 34, Uzice, Serbia \\ ${ }^{3}$ University of Niš, Faculty of Mechanical Engineering, Niš, Serbia \\ ${ }^{4}$ University of Belgrade, Institute of Physics Belgrade - National Institute of the Republic of Serbia, Belgrade- \\ Zemun, Serbia \\ E-mail: miroslava.jordovic-pavlovic@vpts.edu.rs
}

\begin{abstract}
In this paper, the influence of the input and output data scaling and normalization on the neural network overall performances is investigated aimed at inverse problem-solving in photoacoustics of semiconductors. The logarithmic scaling of the photoacoustic signal amplitudes as input data and numerical scaling of the sample thermal parameters as output data are presented as useful tools trying to reach maximal network precision. Max and min-max normalizations to the input data are presented to change their numerical values in the dataset to common scales, without distorting differences. It was demonstrated in theory that the largest network prediction error of all targeted parameters is obtained by a network with non-scaled output data. Also, it was found out that the best network prediction was achieved with min-max normalization of the input data and network predicted output data scale within the range of [110]. Network training and prediction performances analyzed with experimental input data show that the benefits and improvements of input and output scaling and normalization are not guaranteed but are strongly dependent on a specific problem to be solved.
\end{abstract}

Keywords: photoacoustic, semiconductors, artificial neural networks, thermal diffusion, thermal expansion, photothermal, inverse problem

\section{Introduction}

It is a well-known fact that data scaling and normalization in neural networks are the techniques usually applied as part of the data preparation process. Their ultimate goal is to change the values of data in the dataset to a common scale without distorting input differences. The main difference, followed in this article, between scaling and normalization is that, in scaling, only the data range is changing, helping one to compare different variables in the same range. In normalization, not only the range but the shape of the data distribution is changed, too, helping one to calculate differences easier avoiding, for example, possible data overlapping. In general, scaling helps one to compare different variables on equal footing and normalization is used trying to improve the network model due to its numerical stability and quality of the training process. Both scaling and 
normalization can be applied in neural networks not only on input (for example, arbitrary signals) but on output data (predicted parameters) as well [1-4].

In photoacoustics (PA), scaling and normalization process is usually used in experimental data analysis for accurate and reliable characterization of the investigated materials, preferably in two cases: 1) to eliminate the influence of the measuring system and 2) to find the differences in photoacoustic signal behavior by detecting small changes that correspond to possible thermal, mechanic and/or electronic parameters variations. In both cases, photoacoustics uses the data set containing two features: amplitudes and phases in the modulation frequency range from $(20-20 \mathrm{k})$ Hz. In the given frequency range the phase varies from (0-360) deg, while the amplitude variations are within the range of $\left(10^{-3}-10^{-8}\right)$ a.u. and lower. It is obvious that these two features are in different ranges [1-8]. Many authors use this difference to define the amplitude of the photoacoustic signal as the main feature which will intrinsically influence more on the final analysis result due to its larger changes, which implicating that amplitudes are a more important predictor in signal analysis than phases [9-18]. But phases are the constitutive part of the signal and they are, also, sensitive to the sample parameters changes, meaning that both features have to be considered as predictors of equal importance. Our experience shows that, within the application of artificial neural networks (ANNs) in photoacoustics, in most cases the usage of only one feature (amplitude or phase) will give the reliable and accurate results of network predictions. This is the reason why, in this article, we will use only amplitudes of the photoacoustic signal as an ANNs input $[9,10]$.

In our previous articles we have shown that the application of ANNs in photoacustics could improve experimental procedures in many ways: better accuracy and precision in investigated sample parameters prediction, better control of the experimental conditions together with approaching to the real-time characterization of the investigated sample, etc [9.10]. Here, in this article, we will try to show why the different types of scaling and normalization procedures of input and output data could be beneficial to the accuracy, precision and numerical stability of the network predicted parameters, and to the process of network training acceleration. To do that logarithmic scaling and min-max and max normalizations will be applied on input data used in the ANNs training process. At the same time, simple numerical scaling will be used for network output data (predicted sample thermal and geometric parameters such as: thermal diffusivity, linear coefficient of thermal expansion, thickness...) to find possible benefits to ANNs performances. Our analysis of training, stability and accuracy of network prediction will rely on the ANNs that are trained with 
or without scaling and/or normalization of input and output data aiming to found their influence to overall network performances.

\section{Photoacoustic theory and experimental response}

The photoacoustic signals are generated as a consequence of the thermal state changes in semiconductors due to the absorption of monochromatic modulated light source having the intensity of $I=I_{0}\left(1+e^{i \omega t}\right)$ ( $I_{0}$ is the incident light amplitude and $\omega=2 \pi f$ where $f$ is the modulation frequency). Using theoretical model of composite piston the photoacoustic signals in semiconductors, so called total PA signals $\delta \tilde{p}_{\text {total }}(f)$ generated by the investigated sample can be presented in the form $[5-8,19-22]$ :

$$
\delta \tilde{p}_{\text {total }}(f)=K_{1} \tilde{T}_{s}(l, f) e^{i\left(\omega t-\frac{\pi}{4}\right)}+K_{2} \alpha_{\mathrm{T}} \frac{R_{s}^{4}}{l^{3}} \int_{-l / 2}^{l / 2} z \tilde{T}_{s}(x, f) d x+K_{3} d_{n} \frac{R_{\mathrm{S}}^{4}}{l^{3}} \int_{-l / 2}^{l / 2} z \delta \tilde{n}_{\mathrm{p}}(x, f) d x
$$

where $K_{\mathrm{i}} i=1,2,3$ are the constants dependent on the thermodynamic state of the gas in the photoacoustic cell and its geometrical properties, $R_{\mathrm{S}}$ and $l$ are the radius and thickness of the sample, respectively, $\alpha_{\mathrm{T}}$ is the coefficient of linear expansion of the sample, $d_{n}$ is the coefficient of electronic deformation, $\delta \tilde{n}_{\mathrm{p}}(x, f)$ is the concentration of minority excess charge carriers and $\tilde{T}_{s}(x, f)$ is the temperature distribution along the sample. Knowing that the solutions of $\tilde{T}_{s}(x, f)$ and $\delta \tilde{n}_{\mathrm{p}}(x, f)$ can be found using complex analysis, Eq.(1) can be presented, also, in the simplified complex form:

$$
\delta \tilde{p}_{\text {total }}(f)=A_{\text {total }}(f) \exp \left[i \phi_{\text {total }}(f)\right]
$$

where $A_{\text {total }}(f)$ is the total signal amplitude and $\phi_{\text {total }}(f)$ is the total signal phase. Usually, measured experimental signals $\tilde{S}_{\text {total }}^{\exp }(f)$ are the combinations of $\delta \tilde{p}_{\text {total }}(f)$ and influence of the 
measuring system $\tilde{H}_{\text {total }}(f)$ (the deviations caused by the microphone and accompanied electronics), written as:

$$
\tilde{S}_{\text {total }}^{\exp }(f)=\tilde{p}_{\text {total }}^{\exp }(f) \cdot \tilde{H}_{\text {total }}(f) \text {. }
$$

To eliminate the influence $\tilde{H}_{\text {total }}(f)$ of the measuring system, correction procedure of $\tilde{S}_{\text {total }}^{\exp }(f)$ was established aiming to find characteristic parameters of the used instruments (microphone and accompanied electronics) and remove $\tilde{H}_{\text {total }}(f)$ from Eq.(3). In such way only $\delta \tilde{p}_{\text {total }}(f)$ remains in the Eq.(3), suitable to fit with Eq.(1). Mentioned fitting procedure will allows one to extract all thermal (for example $D_{\mathrm{T}}, \alpha_{\mathrm{T}}, \tilde{T}_{s}(x, f)$ ) and electronic (for example $\delta \tilde{n}_{\mathrm{p}}(x, f), d_{n}$ ) parameters needed for sample material characterization.

In this article, theoretically obtained $\delta \tilde{p}_{\text {total }}(f)$ signal amplitudes (Eq.(1-2)) are used to form the network training database. After signal correction procedure (Eq.(3)), obtained $\delta \tilde{p}_{\text {total }}^{\exp }(f)$ amplitudes are presented to the networks and used for "intelligent characterization" of semiconductors (ANNs prediction of the sample parameters).

\section{Neural networks model design}

As it was explained in [9,10], we used the simplest model in creating our network, the so-called "feed-forward network", having an input, one hidden and the output layer. The input layer consists of 72 neurons (amplitudes) following the standard number of modulation frequencies used in our experiments. The hidden layer consists of 50 neurons, following the criteria that the number of neurons is less than half the size of the input layer. The output layer consists of 3 neurons, each corresponding to the number of predictions we want to make (typical investigated sample thermal $-D_{\mathrm{T}}, \alpha_{\mathrm{T}}$ and geometric - $l$ parameters). The sigmoid activation function (Logistic Sigmoid) is used to convert the input into a more useful output with values between 0 and 1 .

Network database consists of 5381 amplitudes (Figure 1.a) of the PA theoretical signals $\delta \tilde{p}_{\text {total }}(f)$ obtained with Eqs.(1-2), changing the sample parameters in the following ranges: 
$D_{\mathrm{T}}=(8.1-9.9) \cdot 10^{-5} \mathrm{~m}^{2} \mathrm{~s}^{-1}, \quad \alpha_{\mathrm{T}}=(2.34-2.86) \cdot 10^{-6} \mathrm{~K}^{-1}$ and $\quad l=(1-10) \cdot 10^{-8} \mathrm{~m} . \quad$ In such way satisfying variety, density and volume of relevant data is achieved. Sometimes, simple amplitude scaling on unity (scaled on 1) is performed (Figure 1.b) to emphasize differences in frequency domain $[9,10]$.
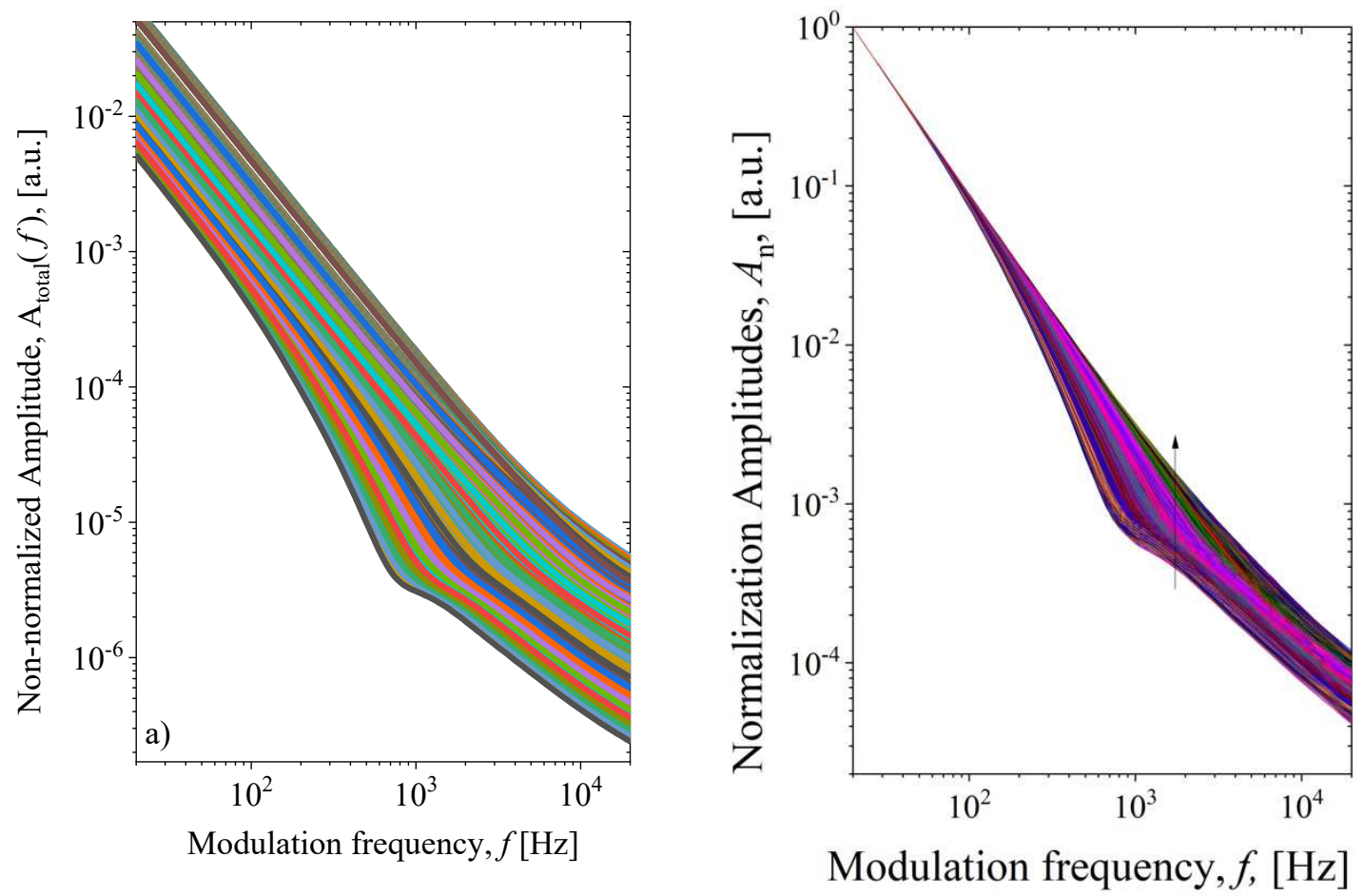

Figure 1. a) Non-scaled amplitude characteristics of the theoretical photoacoustic signals $[9,10]$ that forms the network training database; b) simple amplitude scaling on unity to emphasize differences in frequency domain. The arrows indicate an increase in the thickness $l$.

Typical supervised learning is used here in network training, applying regression technique for output data prediction together with back-propagation algorithm that assures satisfying network prediction accuracy finding optimal values of weights. The training procedure was performed with or without input signal amplitudes and output parameters normalization or scaling. To evaluate our network model the test of its prediction accuracy on data from the training set which was not used at all during training was performed. Networks trained in mentioned way are used to predict thermal parameters of experimentally obtained PA signals from three n-type silicon samples with different thicknesses. 


\section{Input and output data scaling and normalization}

It is obvious from Figure 1 that scaling is needed due to the large changes in amplitude values (few orders of magnitude). The scaling is usually performed with logarithmic function which satisfies the criteria to keep amplitude distribution unchanged in frequency domain. Also, max and minmax normalization are used to change the amplitude distribution in the frequency range and, at the same time, to rescale amplitudes to the ranges of $(0,1]$ and $[0,1]$, respectively.

Scaling of PA amplitudes $A(f)$ from the training base (Figure 1.a) applied here is performed using the logarithmic function (log scaled) based on the Bode plots, having the form

$$
A_{\log }\left(f_{i}\right)=20 \cdot \log A\left(f_{i}\right), \quad i=1,2, \ldots 72
$$

and the results based on the $\mathrm{Eq}(4)$ are presented in Figure 2.a. As it can be seen, logarithmic function changes the amplitude values to a common scale, within the range of phase values. Comparing Fig.1.a and Fig.2.a one can see that this type of scaling does not change the type of $A(f)$ distribution in the investigated modulation frequency range of $20 \mathrm{~Hz}$ to $20 \mathrm{kHz}$.
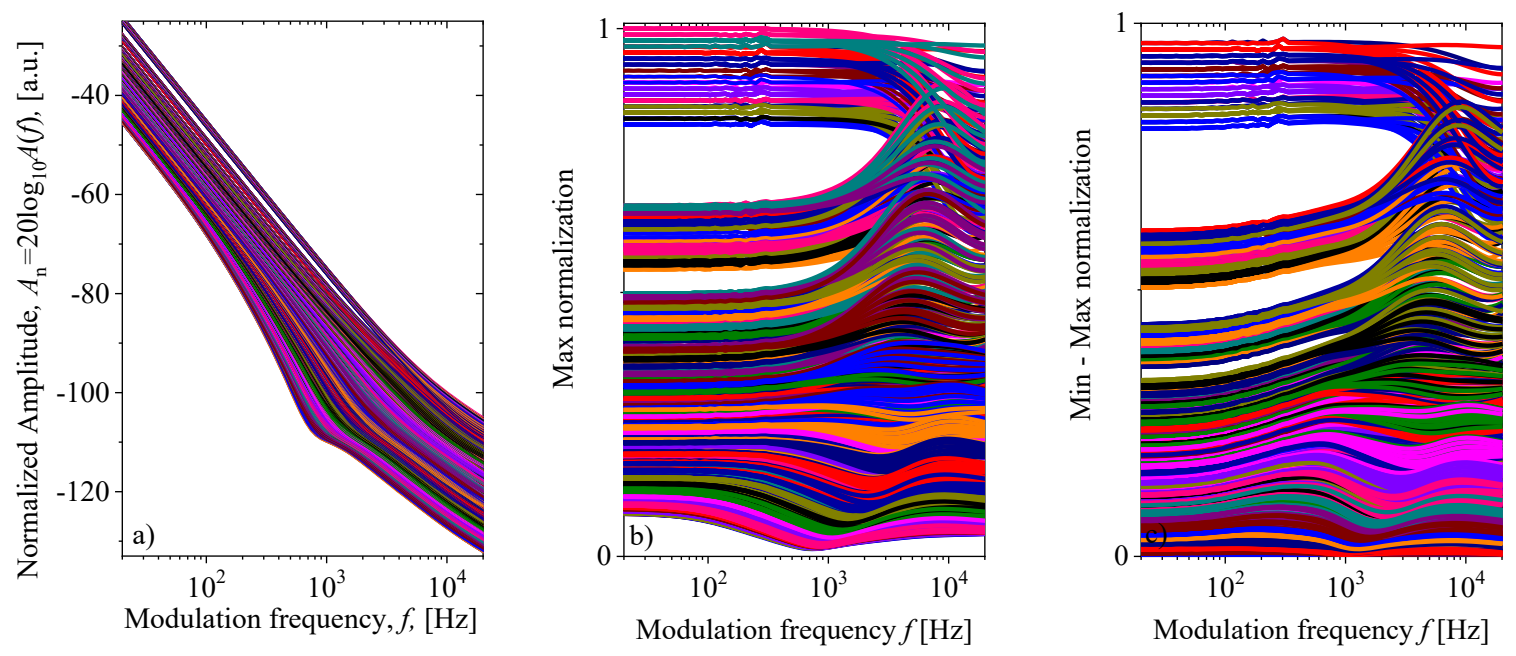

Figure 2. Databases for neural network training: amplitudes a. scaled by logarithmic function, and b. rescaled by max and c. min-max normalization. 
First type of normalization applied on $A(f)$ is the normalization to the maximum absolute value (max norm) of the base frequency vectors (Figure 2.b), defined as [23,24]:

$$
A_{\max }\left(f_{i}\right)=\frac{A\left(f_{i}\right)}{\left|\max \left(A_{j}\left(f_{i}\right), j=1, \ldots, 5381\right)\right|}, i=1,2, \ldots 72 .
$$

This type of normalization gives amplitude values within the range of $0<A_{\max }\left(f_{i}\right) \leq 1$ and changes the amplitude distribution in the given modulation frequency range of $20 \mathrm{~Hz}-20 \mathrm{kHz}$.

Second normalization applied on $A(f)$ is the min-max normalization (min-max norm) which rescales the range of the amplitudes to [0,1] (Figure 2.c), using $[25,26]$ :

$$
A_{\min \max }\left(f_{i}\right)=\frac{A\left(f_{i}\right)-A_{\min }}{A_{\max }-A_{\min }}, i=1,2, \ldots 72 .
$$

where $A_{\max }=\max \left(A_{j}\left(f_{i}\right)\right), j=1, \ldots, 5381$ and $A_{\min }=\min \left(A_{j}\left(f_{i}\right)\right), j=1, \ldots, 5381$.

This type of normalizations (Eq. (6)) is depicted in Figure 2.c, simultaneously changing the type of $A(f)$ distribution in the investigated modulation frequency range.

It is assumed that both types of input data normalization could lead to better network predictions in critical areas $(>1 \mathrm{kHz})$ where some signal overlapping exists. Then, it can be said that databases in the range 0 to 1 (Figure 2.b and c) are more acceptable for machine learning because it is easier to form a weight matrix using numerical values of a certain smaller range. The slight difference that exists in max and min-max normalizations is due to the normalization of the maximum value of the signal at a certain frequency, while the normalization of min-max is the normalization to the range $[0,1]$.

Two ANNs with different ways of scaling the output data are formed to analyze the influence of scaling on the network's training performances. As input parameters of both ANNs, the unchanged (non-scaled and non-normalized) PA signal amplitudes (Figure 1.a) are used. As we said earlier, the data of the output vectors are three parameters of the sample, given in the following ranges: $D_{\mathrm{T}}=(8.1-9.9) \cdot 10^{-5} \mathrm{~m}^{2} \mathrm{~s}^{-1}, \alpha_{\mathrm{T}}=(2.34-2.86) \cdot 10^{-6} \mathrm{~K}^{-1}$, and $l=(1-10) \cdot 10^{-4} \mathrm{~m}$. For the first neural network (NN1) with non-scaled output, we set the thermal diffusivity range as the 
smallest data in the output vector $(0.81-0.99)$; the linear expansion in the range $(2.34-2.86)$; the thickness in the range $(100-1000)$. For the second neural network (NN2), we scaled the output data in the range of values from 1 to $10:(8.10-9.90) ;(2.34-2.86) ;(1-10)$.

\section{Results and discussion}

\subsection{Neural networks with non-scaled and scaled output}

To test the network training performances (a plot of Mean Squared Error (MSE) vs Epochs), we train NN1 and NN2 networks. The results of obtained network training performances are given in Table 1. It is obvious that NN2 networks shows much better results in network training performances, having larger number of epochs but same training time as in the case of NN1.

Table 1. Training performance of two neural networks with non-scaled input: the first with non-scaled output NN1, the second with scaled output NN2.

\begin{tabular}{cc}
\hline Type of NN & Performance \\
\hline NN1 non-scaled output & MSE 0.059084 at 809 epoch, training time 4 h $30 \mathrm{~min}$. \\
\hline NN2 scaled output & MSE 0.000037822 at 1000 epoch, training time 4 h 30 min. \\
\hline
\end{tabular}

Network prediction accuracy is tested using maximal and average relative errors obtained comparing network prediction and parameters values used to form the theoretical amplitudes. The tests are performed on signals not presented to the network during the training: 110 randomly selected signals from the training database, and 24 randomly selected signals out of the training base (with random parameters within the range of parameter changes).

Table 2. Maximal and average (\%) relative prediction error of two neural networks with differently scaled output layer on independent amplitude test.

\begin{tabular}{ccccccc}
\hline 110 & \multicolumn{2}{c}{ max (\%) relative error } & \multicolumn{3}{c}{ average (\%) relative error } \\
\hline Type of NN & $D_{\mathrm{T}}$ & $\alpha_{\mathrm{T}}$ & $l$ & $D_{\mathrm{T}}$ & $\alpha_{\mathrm{T}}$ & $l$ \\
\hline NN1 & 11.8496 & 7.2159 & 0.4632 & 3.9795 & 1.7724 & 0.0632 \\
\hline NN2 & 0.4824 & 0.2173 & 0.4756 & 0.0661 & 0.0574 & 0.0720 \\
\hline
\end{tabular}

Table 3. Maximal and average (\%) relative error of two neural networks with differently scaled output layer data in amplitude prediction of randomly selected parameters in the range of changes of parameters.

\begin{tabular}{ccccccc}
\hline 24 & \multicolumn{3}{c}{$\max (\%)$ relative error } & \multicolumn{3}{c}{ average (\%) relative error } \\
\hline Type of NN & $D_{\mathrm{T}}$ & $\alpha_{\mathrm{T}}$ & $l$ & $D_{\mathrm{T}}$ & $\alpha_{\mathrm{T}}$ & $l$ \\
\hline $\mathrm{NN} 1$ & 9.2781 & 8.0963 & 3.3938 & 3.2013 & 1.8035 & 0.3448 \\
\hline NN2 & 5.7019 & 6.5365 & 4.5952 & 0.5167 & 0.3886 & 0.4285 \\
\hline
\end{tabular}


The results presented in Tables 1-3 indicate that the scaling of output data is beneficial not only in the case of network training performance but also for network prediction performances, as well. Only the results obtained for the sample thickness prediction deviate from the expected ones (Table 2 and 3). This could have been expected knowing that the theoretical model (Eq.(1)) considers thickness as a parameter obtained by fitting the larger error so the thickness prediction results can vary on a much larger scale than $D_{\mathrm{T}}$ and $\alpha_{\mathrm{T}}$.

\subsection{Neural networks with non-scaled, scaled and normalized input}

Based on the results presented in the previous paragraph NN2 network seems to be more acceptable than NN1 so, our choice for further theoretical analysis will be the NN2. Its training performances are analyzed using different PA signal amplitudes $A(f)$ data as an input in the form of non-scaled (Figure 1.a), scaled on 1 (Figure 1.b), logarithmically scaled (Figure 2.a), max (Figure 2.b) and min-max normalized (Figure 2.c). The results of such analysis are shown in Table 4.

Table 4. The NN2 performances with different input scalings and normalizations.

\begin{tabular}{cc}
\hline type of input normalization & performance \\
\hline non-scaled & 0.000037822 \\
\hline scaled on 1 & 0.00015258 \\
\hline log scaled & 0.000045951 \\
\hline max normalization & 0.0000041492 \\
\hline min-max normalization & 0.00000041263 \\
\hline
\end{tabular}

It is obvious that the worst result in Table 4 is network training with data scaled on unity. Logarithmic scaling significantly improves network performances, max normalization gives better results, while max-min normalization shows the best performances. In general, the presented analysis shows that data normalization is beneficial in terms of network training performances.

The standard NN2 network prediction accuracy test is performed with independent signals extracted from the amplitude base before training $[9,10]$. The results presented in Table 5 show small relative (\%) errors for all types of normalization. This test confirms that the best prediction accuracy was achieved with min-max input normalization. 
Table 5. Maximal and average (\%) relative prediction error on the test of the independent signal amplitudes extracted before network training.

\begin{tabular}{lcccccc}
\hline type of normalization & \multicolumn{3}{c}{ max relative error \% } & \multicolumn{3}{c}{ average relative \% error } \\
\hline parameters & $D_{\mathrm{T}}$ & $\alpha_{\mathrm{T}}$ & $l$ & $D_{\mathrm{T}}$ & $\alpha_{\mathrm{T}}$ & $l$ \\
\hline non-scaled & 0.4824 & 0.2173 & 0.4757 & 0.0661 & 0.0574 & 0.0720 \\
\hline scaled on 1 & 1.1474 & 1.0572 & 1.0963 & 0.1417 & 0.1234 & 0.1700 \\
\hline logarithmic normalization & 0.3472 & 0.2289 & 0.3882 & 0.0661 & 0.0729 & 0.0679 \\
\hline max normalization & 0.1264 & 0.1998 & 0.1939 & 0.0254 & 0.0352 & 0.0265 \\
\hline min-max normalization & 0.0322 & 0.0770 & 0.0605 & 0.0068 & 0.0185 & 0.0084 \\
\hline
\end{tabular}

\subsection{Application on experimental signals}

All previous considerations and tests of network training performances and their prediction accuracy have been done on theoretical signals used for precise determination of changes in $D_{\text {T, }}$ $\alpha_{\mathrm{T}}$ and $l$. Interesting results are obtained by analyzing the network prediction accuracy of experimental samples with different thicknesses (Table 6). The predictions of the thinnest sample are most accurate in the case of NN1 network, while in the case of thicker samples the better predictions are obtained with the NN2. Obviously, NN2 output scaling matches the scale of precise network prediction. This logic does not work with thinner samples where NN1 non-scaled output variables result in a more precise parameters prediction. As a conclusion based on Table 6, one can say that scaling the output data is useful in the case of PA signal amplitudes processing originating from thicker samples. Approaching the thinner ones, output scaling loses its importance. In our case (Figure 1), since we work in most cases with thick samples, the choice of an NN2 network for further experimental amplitudes analysis proves to be a rational solution.

Table 6. Parameter prediction $D_{\mathrm{T}}^{\mathrm{ANN}}, \alpha_{\mathrm{T}}^{\mathrm{ANN}}$ and $l^{\mathrm{ANN}}$ of amplitude neural networks NN1 and NN2. The relative (\%) error of prediction of parameters of individual samples is given. Sample no.1 is $830 \mu \mathrm{m}$, sample no. 2 is $417 \mu \mathrm{m}$ and 3 is $128 \mu \mathrm{m}$.

\begin{tabular}{lccccccccc}
\hline $\begin{array}{l}\text { Rel error } \\
(\%)\end{array}$ & \multicolumn{3}{c}{$\begin{array}{c}\text { Sample no.1 } \\
830 \mu \mathrm{m}\end{array}$} & \multicolumn{3}{c}{$\begin{array}{c}\text { Sample no.2 } \\
417 \mu \mathrm{m}\end{array}$} & \multicolumn{3}{c}{$\begin{array}{c}\text { Sample no. 3 } \\
128 \mu \mathrm{m}\end{array}$} \\
\hline parameters & $D_{\mathrm{T}}^{\mathrm{ANN}}$ & $\alpha_{\mathrm{T}}^{\mathrm{ANN}}$ & $l^{\mathrm{ANN}}$ & $D_{\mathrm{T}}^{\mathrm{ANN}}$ & $\alpha_{\mathrm{T}}^{\mathrm{ANN}}$ & $l^{\mathrm{ANN}}$ & $D_{\mathrm{T}}^{\mathrm{ANN}}$ & $\alpha_{\mathrm{T}}^{\mathrm{ANN}}$ & $l^{\mathrm{ANN}}$ \\
\hline $\mathrm{NN} 1$ & 0.6556 & 0.3590 & 0.0133 & 0.6035 & 0.9553 & 0.0831 & 1.9162 & 1.6576 & 2.1096 \\
\hline $\mathrm{NN} 2$ & 0.0555 & 0.0421 & 0.0111 & 0.1290 & 0.0263 & 0.0592 & 11.7065 & 5.7570 & 11.5476 \\
\hline
\end{tabular}


NN2 prediction accuracy test was applied on experimental signals as well, and the results are presented in Table 7. The benefits of the input data normalization of the NN2 network can be seen, also, but not as obvious as with theoretical signals. The reason for that lies in the fact that the experimental signals are neither in frequency nor parameters changing steps as theoretical signals are. This is the reason why one can expect larger errors and more diversity of NN2 parameters prediction accuracy obtained with experimental signals. Largest errors are obtained mostly with non-scaled inputs.

Table 7. The NN2 parameter prediction $D_{\mathrm{T}}^{\mathrm{ANN}}, \alpha_{\mathrm{T}}^{\mathrm{ANN}}$ and $l^{\mathrm{ANN}}$ with non-scaled, scaled and normalized inputs on experimental photoacoustic signals. The relative (\%) error of prediction of parameters of individual samples is given. Sample no. 1 is $830 \mu \mathrm{m}$, sample no. 2 is $417 \mu \mathrm{m}$ and no. 3 is $128 \mu \mathrm{m}$.

\begin{tabular}{lccccccccr}
\hline Rel error (\%) & \multicolumn{3}{c}{ Sample no.1 } & \multicolumn{3}{c}{ Sample no.2 } & \multicolumn{3}{c}{ Sample no. 3 } \\
\hline \multicolumn{1}{c}{ parameters } & $D_{\mathrm{T}}^{\text {ANN }}$ & $\alpha_{\mathrm{T}}^{\text {ANN }}$ & $l^{\text {ANN }}$ & $D_{\mathrm{T}}^{\text {ANN }}$ & $\alpha_{\mathrm{T}}^{\text {ANN }}$ & $l^{\text {ANN }}$ & $D_{\mathrm{T}}^{\text {ANN }}$ & $\alpha_{\mathrm{T}}^{\text {ANN }}$ & $l^{\text {ANN }}$ \\
\hline non-scaled & 0.0555 & 0.0421 & 0.0111 & 0.1290 & 0.0263 & 0.0592 & 11.7065 & 5.7570 & 11.5476 \\
\hline scaled on 1 & 0.0367 & 0.0812 & 0.0037 & 0.1120 & 0.0635 & 0.0011 & 2.8903 & 2.0515 & 2.9798 \\
\hline log scaled & 0.0450 & 0.0387 & 0.0204 & 0.0610 & 0.0339 & 0.0130 & 5.8210 & 2.3487 & 6.2749 \\
\hline max norm & 0.0672 & 0.0278 & 0.0005 & 0.0774 & 0.0316 & 0.0116 & 1.2337 & 0.1819 & 2.3816 \\
\hline min-max norm & 0.0366 & 0.0489 & 0.0188 & 0.0651 & 0.0124 & 0.0031 & 2.3220 & 0.3906 & 3.1880 \\
\hline
\end{tabular}

\section{Conclusion}

In this article, the influence of different input and output data scaling and normalization on overall neural network performances in photoacoustics is presented.

In theory, simple numerical scaling is applied on network prediction parameters (diffusivity, coefficient of thermal expansion, thickness) as output data. This kind of scaling was found to be beneficial in the terms of network training and prediction and it was kept throughout the whole analysis. Various scaling and normalization methods (logarithmic scaling, scaling on unity, max and min-max normalization) were applied to the photoacoustic signal amplitudes as input predictors of equal importance compared to the signal phase. It was found that min-max amplitude normalization shows the best results in network training and prediction accuracy.

In the experiment, the results of network input scaling and/or normalization are not unambiguous. In general, each method has pros and cons based on the specific problem to be solved and one can decide based on the problem which scaling or normalization method is best suitable 
for the problem. The results obtained here with the silicon samples of different thicknesses suggesting that max normalization of input and non-scaled output data are the best choice to reach the highest quality of network overall performances in the case of thicker samples. In the case of thinner samples, various scaling and normalization methods can be only partially beneficial for network overall performances. As a principal conclusion, there is no universal input data normalization method that can be chosen in advance to improve network training performances and prediction accuracy.

\section{Acknowledgments}

This work was supported by the Ministry of Education, Science and Technological Development of the Republic of Serbia, contract number 451-03-09/2021-14/200017.

\section{REFERENCES}

[1] Balderas-López JA 2006 Photoacoustic signal normalization method and its application to the measurement of the thermal diffusivity for optically opaque materials, Review of Scientific Instruments 77, 064902;

https://doi.org/10.1063/1.2209951

[2] Barros Melo WL, Faria RM 1995 Photoacoustic procedure for measuring thermal parameters of transparent solids, Appl. Phys. Lett. 67, 3892 https://doi.org/10.1063/1.115308

[3] Govorkov S, Ruderman W, Horn MW, Goodman RB, and Rothschild M 1997 A new method for measuring thermal conductivity of thin films, Review of Scientific Instruments 68, 3828 (1997); https://doi.org/10.1063/1.1148035

[4] Balderas-Lo' pez JA, Mandelis A, and Garci'a JA 2002 Normalized photoacoustic techniques for thermal diffusivity measurements of buried layers in multilayered systems, Journal of Applied Physics 92, 3047 (2002); https://doi.org/10.1063/1.1500784

[5] Todorovic DM, Nikolic PM, Dramicanin MD, Vasiljevic DG, and Ristovski ZD 1995 Photoacoustic frequency heat transmission technique: Thermal and carrier transport parameters measurements in silicon, J. Appl. Phys. 78, 5750 https://doi.org/10.1063/1.359637

[6]Calderón A, Muñoz Hernández RA, and Tomás SA, Cruz-Orea A and Sánchez Sinencio F 1998 Method for measurement of the thermal diffusivity in solids: Application to metals, semiconductors, and thin materials, Journal of Applied Physics 84, 6327 https://doi.org/10.1063/1.368957

[7] Dramićanin MD, Nikolić PM, Ristovski ZD, Vasiljević DG, and Todorović DM 1995 Photoacoustic investigation of transport in semiconductors: Theoretical and experimental study of a Ge single crystal, Physical Review B, 51, 14226 https://doi.org/10.1103/PhysRevB.51.14226

[8] Todorović DM, Rabasović MD, Markushev DD, Sarajlic M 2014 Photoacoustic elastic bending in thin filmsubstrate system: Experimental determination of the thin film parameters. J Appl Phys 116053506 https://doi.org/10.1063/1.4890346

[9] Djordjevic KLj, Markushev DD, Ćojbašić Ž M, Galović S P 2019 Photoacoustic measurements of the thermal and elastic properties of n-type silicon using neural networks, Silicon, Springer DOI: 10.1007/s12633-019-00213-6 
[10] Djordjevic KLj, Markushev DD, Ćojbašić ŽM, Galović SP 2020 Inverse problem solving in semiconductor photoacoustics by neural networks, INVERSE PROBL SCI EN https://doi.org/10.1080/17415977.2020.1787405

[11] Sola J and Sevilla J 1997 Importance of input data normalization for the application of neural networks to complex industrial problems in IEEE Transactions on Nuclear Science, vol. 44, no. 3, pp. 1464-1468 doi: 10.1109/23.589532.

[12] Jian Jin, Ming Li , and Long Jin 2015 Data Normalization to Accelerate Training for Linear Neural Net to Predict Tropical Cyclone Tracks, Mathematical Problems in Engineering ID 931629 https://doi.org/10.1155/2015/931629

[13] Kim D 2007 Normalization methods for input and output vectors in backpropagation neural networks, Taylor\&Francis Online, 161-171 1998 //doi.org/10.1080/00207169908804800

[14] Ren M, Liao R, Urtasun R, Sinz FH, Zemel RS 2017 NORMALIZING THE NORMALIZERS: COMPARING AND EXTENDING NETWORK NORMALIZATION SCHEMES Published as a conference paper at ICLR

[15] Thompson JA, Tan J, Greene CS, Cross-platform normalization of microarray and RNA-seq data for machine learning applications. PeerJ 4:e1621 https://doi.org/10.7717/peerj.1621

[16] Ma W, Liu Z, Kudyshev ZA et al.2020 Deep learning for the design of photonic structures. Nat. Photonics https://doi.org/10.1038/s41566-020-0685-y

[17] Arridge S, Maass P, Öktem O and Schönlieb C 2019 Solving inverse problems using data-driven models. Acta Numerica, 28, 1-174. doi:10.1017/S0962492919000059

[18] Yahyaoui's I,Yahyaoui I, Yumuşak N 2018Machine Learning Techniques for Data Classification. Vol 2: Biomass, Fuel Cells, Geothermal Energies, and Smart Grids 441-450 https://doi.org/10.1016/B978-0-12-813185$\underline{5.00009-7}$

[19] Mandelis A, Hess P 2000 Semiconductors and Electronic Materials, Vol. IV in the Series: Progress in Photothermal and Photoacoustic Science and Technology (SPIE Press, Bellingham, 2000) ISBN: 9780819435064

[20] Markushev DD, Ordonez-Miranda J, Rabasovic MD, Galovic S, Todorovic DM, Bialkowski SE 2015 Effect of the absorption coefficient of aluminium plates on their thermoelastic bending in photoacoustic experiments J. Appl. Phys. 117, 245309 (2015) https://doi.org/10.1063/1.4922718

[21] Markushev DK, Markushev DD, Aleksic SM, Pantic DS, Galovic SP, Todorovic DM, and Ordonez-Miranda J 2019 Effects of the photogenerated excess carriers on the thermal and elastic properties of n-type silicon excited with a modulated light source: Theoretical analysis, J. Appl. Phys, 126, 185102 doi: 10.1063/1.5100837

[22] Markushev DK, Markushev DD, Galović S, Aleksić S, Pantić DS, Todorović DM 2018 The surface recombination velocity and bulk lifetime influences on photogenerated excess carrier density and temperature distributions in n-type silicon excited by a frequency-modulated light source, FU Elec Energ $\underline{\mathbf{3 1}}(2) \quad 313 \quad$ - 328 https://doi.org/10.2298/FUEE1802313M

[23] Jordovic Pavlovic MI, Markushev DD, Kupusinac AD, et al. 2020 Deep Neural Network Application in the PhaseMatch Calibration of Gas-Microphone Photoacoustics. Int J Thermophys 41, 73 https://doi.org/10.1007/ s10765-020$\underline{02650-7}$

[24] Jordovic-Pavlovic MI, Kupusinac AD, Djordjevic KLj et al. 2020 Computationally intelligent description of a photoacoustic detector. Opt Quant Electron 52, 246 https://doi.org/10.1007/s11082-020-02372-y

[25] Furundzic D, Djordjevic M and Bekic AJ 1998 Neural networks approach to early breast cancer detection, J SYST ARCHITECT, 44, 8, 617-633 https://doi.org/10.1016/S1383-7621(97)00067-2 
[26] Furundzic D, Stankovic S, Jovicic S, Punisic S, Subotic M 2017 Distance based resampling of imbalanced classes: With an application example of speech quality assessment, Eng. Appl. Artif. Intell, 64, 440-461 https://doi.org/10.1016/j.engappai.2017.07.001

\section{Declarations}

All manuscripts must contain the following sections under the heading 'Declarations'.

If any of the sections are not relevant to your manuscript, please include the heading and write 'Not applicable' for that section.

To be used for all articles, including articles with biological applications

Funding - Not applicable

Conflicts of interest/Competing interests - Not applicable

Availability of data and material - Not applicable

Code availability - Not applicable

Authors' contributions - Not applicable

Additional declarations for articles in life science journals that report the results of studies

involving humans and/or animals

Ethics approval - Not applicable

Consent to participate - Not applicable

Consent for publication - Not applicable 